\title{
A model approach to modelling
}

\author{
The work by Roberto Car and Michele Parrinello on ab initio molecular dynamics published 25 years ago \\ has had a huge impact on fundamental science and applications in a wide range of fields.
}

When Roberto Car and Michele Parrinello
started collaborating in the early 1980s,
electronic structure calculations and
molecular dynamics (MD) simulations of
atomic configurations were virtually isolated
from each other. But Car and Parrinello
combined their individual expertise to create
a method that bridged the gap between the
two communities and changed the approach
to materials modelling in a radical way ${ }^{1}$.
The Car-Parrinello ab initio molecular
dynamics method was conceived and
developed within the theoretical physics
community in Trieste, in a period in which
SISSA (Scuola Internazionale Superiore
di Studi Avanzati) was still a very young
institute and was practically overlapping with the well-established International Centre of Theoretical Physics (ICTP). The presence of many bright physicists and the continuous flow of international visitors made the atmosphere highly stimulating. Discussions went on virtually without interruption, even during the occasional swims around the shores of the Miramare Castle (pictured), only a few steps away from the ICTP building. Car's background in density functional theory (DFT) and extreme enthusiasm was perfectly complemented by Parrinello's knowledge of statistical mechanics and molecular dynamics, in addition to his reflective attitude, resulting in an ideal combination of personality and scientific expertise.

The paper reporting the Car-Parrinello method $^{1}$ was published in November 1985, and on the occasion of its twenty-fifth anniversary we explore the origin of the work and its long-lasting impact.

What emerges from the Interviews with the two scientists ${ }^{2,3}$ is that the main motivation for their efforts were the limitations suffered by both DFT and classical MD, which effectively restricted their application to the realistic simulation of condensed matter at finite temperature to specific cases. Pure DFT was mainly applicable to the electronic structures of ordered and homogenous systems. On the other hand, the forces between atoms used in MD did not take into account the fact that the electronic potentials varied with the atomic movement during the progress of a simulation. By using DFT to calculate the potential 'felt' by atoms and letting such potential evolve with each step of the

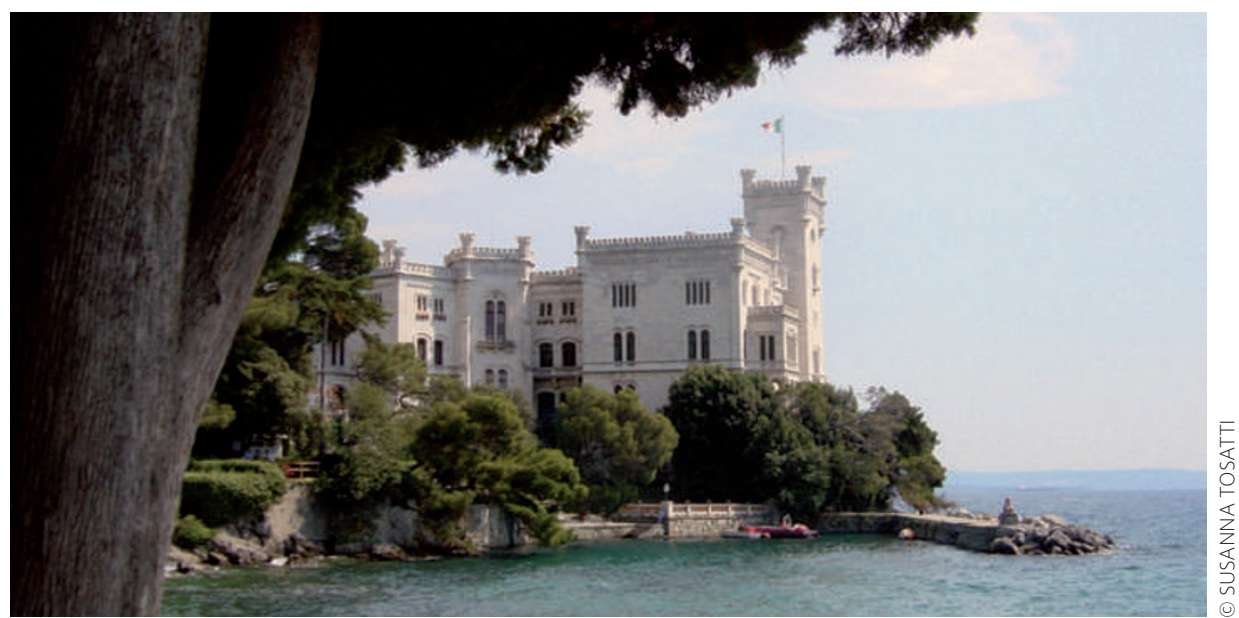

simulation, the Car-Parrinello method allowed a much wider range of disordered and therefore more realistic materials systems to be studied. This was beautifully demonstrated by applying the method to amorphous silicon, which led to results on both the atomic structure and the electronic properties that were in very good agreement with experimental observations ${ }^{4}$. More generally, the ability to follow the evolution of the electronic potential has allowed important studies of chemical reactions occurring, for example, in liquids and large biomolecules.

In his Commentary ${ }^{5}$, besides recalling the fundamental concepts behind the Car-Parrinello method, Jürgen Hafner describes the highly inspirational role that the approach had in stimulating the creation of a series of first-principles computational codes that are still used widely today. A special role for these codes is their application to phenomena that are difficult to study experimentally, such as those occurring at high pressure or during complex and fast chemical reactions.

An aspect of these codes that is not always appreciated is their importance in industrial applications. The CASTEP code ${ }^{6}$, conceived and developed by Mike Payne and collaborators at the University of Cambridge, is a perfect example. Since its commercialization, it has been the highest source of revenue for the university in the physical sciences. The code is the fundamental part of the software package 'Materials Studio', developed and commercialized by Accelrys, with the aim of providing a powerful tool to generic users with no specific knowledge of code writing. According to Gerhard Goldbeck-Wood, director of product marketing for Materials Studio, the software package is used by chemical companies that work in fields such as catalysis or high- $k$ dielectrics, to name just a few. Most importantly an essential aim of these types of code is their use in feasibility tests, which has substantial economical benefits.

Unlike the discovery of a new molecule or the observation of a new phenomenon, it is difficult to appreciate the success that a computational technique is likely to have when it is first introduced. After a quarter of a century however, it is clear that the Car-Parrinello method has been ground-breaking within the field of computational materials science and has had an enormous impact on fundamental science and applications in a wide range of fields, from solid-state materials physics to chemistry and biology. Still, the approach was originally conceived by two scientists motivated solely by their passion for science and their desire to understand nature, with no specific agenda with regard to the possible commercial value of their work. This should send a strong message to decision makers in charge of funding research.

\footnotetext{
References

1. Car, R. \& Parrinello, M. Phys. Rev. Lett. 55, 2471-2474 (1985).

2. Nature Mater. 9, 693-694 (2010).

3. Nature Mater. 9, 694-695 (2010).

4. Car, R. \& Parrinello, M. Phys. Rev. Lett. 60, 204-207 (1988).

5. Nature Mater. 9, 690-692 (2010).

6. www.castep.org
} 